\title{
Quantitative Redundancy in Partial Implications
}

\author{
José L. Balcázar* \\ Department of Computer Science \\ Universitat Politècnica de Catalunya \\ jose.luis.balcazar@upc.edu
}

August 26, 2018

\begin{abstract}
We survey the different properties of an intuitive notion of redundancy, as a function of the precise semantics given to the notion of partial implication.

The final version of this survey will appear in the Proceedings of the Int. Conf. Formal Concept Analysis, 2015.
\end{abstract}

\section{Introduction}

The discovery of regularities in large scale data is a multifaceted current challenge. Each syntactic mechanism proposed to represent such regularities opens the door to wide research questions. We focus on a specific sort of regularities sometimes found in transactional data, that is, data where each observation is a set of items, and defined in terms of pairs of sets of items.

Syntactically, the fact that this sort of regularity holds for a given pair $(X, Y)$ of sets of items is often denoted as an implication: $X \rightarrow Y$. However,

*Partially supported by project BASMATI (TIN2011-27479-C04-04) of Programa Nacional de Investigación (Ministerio de Ciencia e Innovación, Spain) and grant 2014SGR 890 (MACDA) from AGAUR, Generalitat de Catalunya. 
whereas in Logic an implication like this is true if and only if $Y$ holds whenever $X$ does, in our context, namely, partial implications and association rules, it is enough if $Y$ holds "most of the times" $X$ does. Thus, in association mining, the aim is to find out which expressions of that sort are valid for a given transactional dataset: for what $X$ and what $Y$, the transactions that contain $X$ "tend to contain" $Y$ as well.

In many current works, that syntax is defined as if its meaning was sufficiently clear. Then, any of a number of "measures of interestingness" is chosen to apply to them, in order to select some to be output by a data analysis process on a particular dataset. Actually, the mere notation $X \rightarrow Y$ is utterly insufficient: any useful perspective requires to endow these expressions with a definite semantics that makes precise how that naïve intuition of "most of the times" is formalized; only then can we study and clarify the algorithmic properties of these syntactical expressions. Thus, we are not really to "choose a measure of interestingness" but plainly to define what $X \rightarrow Y$ means, and there are many acceptable ways of doing this.

This idea of a relaxed implication connective is a relatively natural concept, and versions sensibly defined by resorting to conditional probability have been proposed in different research communities: a common semantics of $X \rightarrow Y$ is through a lower bound on its "confidence", the conditional probability of $Y$ given $X$. This meaning appears already in the "partial implications" of [27] (actually, "implications partielles", with confidence christened there "prècision"). Some contributions based on Mathematical Logic develop notions related to these partial implications defined in terms of conditional probability: see [18. However, it must be acknowledged that the contribution that turned on the spotlights on partial implications was [3] and the improved algorithm in [4]: the proposal of exploring large datasets in search for association rules of high support and confidence has led to huge amounts of research since. Association rules are partial implications that impose the additional condition that the consequent is a single item.

Three of the major foci of research in association rules and partial implications are as follows. First, the quantity of candidate itemsets for both the antecedent $X$ and, sometimes, the consequent $Y$ grows exponentially with the number of items. Hence, the space to explore is potentially enormous: on real world data, very soon we run already into billions of candidate antecedents. Most existing solutions are based on the acceptance that, as not all of them can be considered within reasonable running times, we make do with those that obey the support constraint ("frequent itemsets"). The 
support constraint combines well with confidence in order to avoid reporting mere statistical artifacts [28] but its major role is to reduce the search space. A wide repertory of algorithms for frequent sets and association rule mining exists by now [1].

Second, many variations have been explored: for instance, cases of more complicated structures in the data and, also, combinations with other machinelearning models or tasks like in [20, 41].

This paper surveys part of a research line that belongs to a third focus: in a vast majority of practical applications, if any partial implication is found at all, it often happens that the search returns hundreds of thousands of them. It is far from trivial to design an associator able to choose well, among them, a handful to show to an impatient user. This is tantamount to modifying the semantics of the partial implication connective, by adding or changing the conditions under which one such expression is deemed valid and is to be reported. Most often, but not always (as we report in Sections 3 and 4) this approach takes the form of "quality evaluations" performed to select which partial implications are to be highlighted for the user. We do not consider this problem solved yet, but deep progresses have been achieved so far; we survey a humble handful of those, where the present author was actively involved. For a wider perspective of all these three aspects of association rule mining, see Part II of [43].

The main link along this paper can be described informally as follows: human intuition, maybe on the basis of our experience with full, standard implications, tends to expect that smaller antecedents are better than larger ones, and larger consequents are better than smaller ones. We call this statement here the central intuition of this paper; many references express, in various variants, this intuition (e.g. [12, 22, 26, 30, 33, 36] just to name a few). This intuition is only partially true in implications, where the GD basis gets to be minimal through the use of subtly enlarged antecedents [17]. This survey paper discusses, essentially, the particular fact that, on partial implications, this intuition is both true and false... as a function, of course, of the actual semantics given to the partial implication connective.

\section{Notation and Preliminary Definitions}

Our datasets are transactional. This means that they are composed of transactions, each of which consists of an itemset with a unique transaction identi- 
fier. Itemsets are simply subsets of some fixed set $\mathcal{U}$ of items. We will denote itemsets by capital letters from the end of the alphabet, and use juxtaposition to denote union, as in $X Y$. The inclusion sign as in $X \subset Y$ denotes proper subset, whereas improper inclusion is denoted $X \subseteq Y$. The cardinality of a set $X$ (either an itemset or a set of transactions) is denoted $|X|$.

\subsection{Partial Implications}

As indicated in the Introduction, the most common semantics of partial implication is its confidence: the conditional empirical probability of the consequent given the antecedent, that is, the ratio between the number of transactions in which $X$ and $Y$ are seen together and the number of transactions that contain $X$. We will see below that this semantics may be somewhat misleading. In most application cases, the search space is additionally restricted by a minimal support criterion, thus avoiding itemsets that appear very seldom in the dataset.

More precisely, for a given dataset $\mathcal{D}$, consisting of $n$ transactions, the supporting set $\mathcal{D}_{X} \subseteq \mathcal{D}$ of an itemset $X$ is the subset of transactions that include $X$. (For the reader familiar with the FP-growth frequent set miner [19], these are the same as their "projected databases", except for the minor detail that, here, we do not remove $X$ from the transactions.)

The support $s_{\mathcal{D}}(X)=\left|\mathcal{D}_{X}\right| / n \in[0,1]$ of an itemset $X$ is the cardinality of the set of transactions that contain $X$ divided by $n$; it corresponds to the relative frequency or empirical probability of $X$. An alternative rendering of support is its unnormalized version, but some of the notions that will play a major role later on are simpler to handle with normalized supports. Now, the confidence of a partial implication $X \rightarrow Y$ is $c_{\mathcal{D}}(X \rightarrow Y)=$ $s_{\mathcal{D}}(X Y) / s_{\mathcal{D}}(X)$ : that is, the empirical approximation to the corresponding conditional probability. The support of a partial implication $X \rightarrow Y$ is $s_{\mathcal{D}}(X \rightarrow Y)=s_{\mathcal{D}}(X Y)$. In both expressions, we will omit the subscript $\mathcal{D}$

whenever the dataset is clear from the context. Clearly, $s_{\mathcal{D}_{Z}}(X)=\frac{\left|\mathcal{D}_{X Z}\right|}{\left|\mathcal{D}_{Z}\right|}=$ $c(Z \rightarrow X)$.

Often, we will assume that $X \cap Y=\emptyset$ in partial implications $X \rightarrow Y$. Some works impose this condition globally; we will mention it explicitly whenever it is relevant, but, generally speaking, we allow $X$ and $Y$ to intersect or, even, to fulfill $X \subseteq Y$. Note that, if only support and confidence are at play, then $c_{\mathcal{D}}(X \rightarrow X Y)=c_{\mathcal{D}}(X \rightarrow Y)$ and $s_{\mathcal{D}}(X \rightarrow X Y)=s_{\mathcal{D}}(X \rightarrow Y)$. 
Of course, in practical terms, after a partial implication mining process, only the part of $Y$ that does not appear in $X$ would be shown to the user.

We do allow $X=\emptyset$ as antecedent of a partial implication: then, its confidence coincides with the support, $c_{\mathcal{D}}(\emptyset \rightarrow Y)=s_{\mathcal{D}}(Y)$, since $s_{\mathcal{D}}(\emptyset)=1$. Allowing $Y=\emptyset$ as consequent as well is possible but turns out not to be very useful; therefore, empty-consequent partial implications are always omitted from consideration. All along the paper, there are occassional glitches where the empty set needs to require separate consideration. Being interested in the general picture, here we will mostly ignore these issues, but the reader can check that these cases are given careful treatment in the original references provided for each part of our discussion.

By $X \Rightarrow Y$ we denote full, standard logical implication; this expression will be called the full counterpart of the partial implication $X \rightarrow Y$.

\subsection{Partial Implications versus Association Rules}

Association rules were defined originally as partial implications $X \rightarrow Y$ with singleton consequents: $|Y|=1$; we abbreviate $X \rightarrow\{A\}$ as $X \rightarrow A$. This decision allows one to reduce association mining to a simple postprocessing after finding frequent sets. Due to the illusion of augmentation, many users are satisfied with this syntax, but, however, more items in the consequent provide more information.

Indeed, in full implications, the expression $(A \Rightarrow B) \wedge(A \Rightarrow C)$ is fully equivalent to $A \Rightarrow B C$, and we lose little by enforcing singleton consequents (equivalently, definite Horn clauses); an exception is the discussion of minimal bases, where nonsingleton consequents allow for canonical bases that are unreachable in the Horn clause syntax [17]. But, in partial implications, $A \rightarrow B C$ says more than the conjunction of $A \rightarrow B$ and $A \rightarrow C$, namely, $B$ and $C$ abound jointly in $\mathcal{D}_{A}$. Whenever possible, $A \rightarrow B C$ is better, being both more economical and more informative. This can be ilustrated by the following example from [8], to which we will return later on.

Example 1 Consider a dataset on $\mathcal{U}=\{A, B, C, D, E\}$ consisting of 12 transactions: 6 of them include all of $\mathcal{U}, 2$ consist of $A B C, 2$ more are $A B$, and then one each of $C D E$ and $B C$. It can be seen that the confidence of both $B \rightarrow A$ and $B \rightarrow C$ is $9 / 11$, whereas the confidence of $B \rightarrow A C$ is 8/11.

Actually, even restricted to association rules, the output of confidencebased associators is often still too large: the rest of this paper discusses 
how to reduce the output with no loss of information, first, and, then, as the outcome is often still too large in practice, we will need to allow for a carefully tuned loss of information.

\section{Redundancy in Confidence-Based Partial Implications}

We start our discussion by "proving correct" our central intuition, that is, providing a natural semantics under which that intuition is correct. For this section, we work under confidence and support thresholds, and it turns out to be convenient to explicitly assume that the left-hand side of each partial implication is included in the right-hand side. We force that inclusion using notations in the style of $X \rightarrow X Y$.

Several references ([2] for one) have considered the following argument: assume that we could know beforehand that, in all datasets, the confidence and support of $X_{0} \rightarrow X_{0} Y_{0}$ are always larger than or equal to those of $X_{1} \rightarrow X_{1} Y_{1}$. Then, whenever we are mining some dataset under confidence and support thresholds, assume that we find $X_{1} \rightarrow X_{1} Y_{1}$ : we should not bother to report as well $X_{0} \rightarrow X_{0} Y_{0}$, since it must be there anyhow, and its presence in the output is uninformative. In a very strong sense, $X_{0} \rightarrow X_{0} Y_{0}$ is redundant with respect to $X_{1} \rightarrow X_{1} Y_{1}$. Irredundant partial implications according to this criterion are called "essential rules" in [2] and representative rules in [21]; we will follow this last term.

Lemma 1 Consider two partial implications, $X_{0} \rightarrow X_{0} Y_{0}$ and $X_{1} \rightarrow X_{1} Y_{1}$. The following are equivalent:

1. The confidence and support of $X_{0} \rightarrow X_{0} Y_{0}$ are larger than or equal to those of $X_{1} \rightarrow X_{1} Y_{1}$, in all datasets: for every $\mathcal{D}, c_{\mathcal{D}}\left(X_{0} \rightarrow X_{0} Y_{0}\right) \geq$ $c_{\mathcal{D}}\left(X_{1} \rightarrow X_{1} Y_{1}\right)$ and $s_{\mathcal{D}}\left(X_{0} \rightarrow X_{0} Y_{0}\right) \geq s_{\mathcal{D}}\left(X_{1} \rightarrow X_{1} Y_{1}\right)$.

2. The confidence of $X_{0} \rightarrow X_{0} Y_{0}$ is larger than or equal to that of $X_{1} \rightarrow$ $X_{1} Y_{1}$, in all datasets: for every $\mathcal{D}, c_{\mathcal{D}}\left(X_{0} \rightarrow X_{0} Y_{0}\right) \geq c_{\mathcal{D}}\left(X_{1} \rightarrow X_{1} Y_{1}\right)$.

3. $X_{1} \subseteq X_{0} \subseteq X_{0} Y_{0} \subseteq X_{1} Y_{1}$.

When these cases hold, we say that $X_{1} \rightarrow X_{1} Y_{1}$ makes $X_{0} \rightarrow X_{0} Y_{0}$ redundant. The fact that the inequality on support follows from the inequality on 
confidence is particularly striking. This lemma can be interpreted as proving correct the central intuition that smaller antecedents and larger consequents are better, by indentifying a semantics of the partial implication connective that makes this true and by pointing out that it is not just the consequent that is to be maximized, but the union of antecedent and consequent. If only consequents are maximized separately, and are kept disjoint from the antecedents, then one gets to a quite more complicated situation discussed below.

Definition 1 Fix a dataset and confidence and support thresholds. The representative rule basis for that dataset at these support and confidence thresholds consists of those partial implications that pass both thresholds in the dataset, and are not made redundant, in the sense of the previous paragraph, by other partial implications also above the thresholds.

Hence, a redundant partial implication is so because we can know beforehand, from the information in the basis, that its confidence is above the threshold. We have:

Proposition 1 (Essentially, from [21].) For a fixed dataset $\mathcal{D}$ and a fixed confidence threshold $\gamma$ :

1. Every partial implication of confidence at least $\gamma$ is made redundant by some representative rule.

2. Partial implication $X \rightarrow Y$ with $X \subseteq Y$ is a representative rule if and only if $c_{\mathcal{D}}(X \rightarrow Y) \geq \gamma$ but there is no $X^{\prime}$ and $Y^{\prime}$ with $X^{\prime} \subseteq X$ and $X Y \subseteq X^{\prime} Y^{\prime}$ such that $c_{\mathcal{D}}\left(X^{\prime} \rightarrow Y^{\prime}\right) \geq \gamma$, except $X=X^{\prime}$ and $Y=Y^{\prime}$.

According to statement (3) in Lemma 1, that last point means that a representative rule is not redundant with respect to any partial implication (different from itself) that has confidence at least $\gamma$ in the dataset. It is interesting to note that one does not need to mention support in this last proposition, the reason being, of course, statement (2) in Lemma 1, The fact that statement (3) implies statement (1) was already pointed out in [2, 21, 31$]$ (in somewhat different terms). The remaining implications are from [7]; see this reference as well for proofs of additional properties, including the fact the representative basis has the minimum possible size among all bases for this notion of redundancy, and for discussions of other related redundancy 
notions. In particular, several other natural proposals are shown there to be equivalent to this redundancy. Also [8] provides further properties of the representative rules. These references discuss as well the connection with a similar notion in [42].

In Example 1, at confidence threshold 0.8, the representative rule basis consists of seven partial implications: $\emptyset \rightarrow C, B \rightarrow C, \emptyset \rightarrow A B, C \rightarrow A B$, $A \rightarrow B C, D \rightarrow A B C E$, and $E \rightarrow A B C D$.

\subsection{Quantitative Evaluation of Non-Redundancy: Con- fidence Width}

Redundancy is a qualitative property; still, it allows for a quantitative discussion. Consider a representative rule $X \rightarrow X Y$ : at confidence $c(X \rightarrow X Y)$, no partial implication makes it redundant. But we could consider now to what extent we need to reduce the confidence threshold in order to find a partial implication that would make this one redundant. If a partial implication of almost the same confidence can be found to make $X \rightarrow X Y$ redundant, then our partial implication is not so interesting. According to this idea, one can define a parameter, the confidence width [6], that, in a sense, evaluates how different is our partial implication from other similar ones. We do not discuss this parameter further, but a related quantity is treated below in Section 6.2.

\subsection{Closure-Aware Redundancy Notions}

Redundancy of one partial implication with respect to another can be redefined as well in a similar but slightly more sophisticate form by taking into account the closure operator obtained from the data (see [15]). Often, this variant yields a more economical basis because the full implications are described by their often very short Guigues-Duquenne basis [17]; see again [7] for the details.

\section{Redundancy with Multiple Premises}

The previous section indicates precisely when "one partial implication follows logically from another". It is natural to ask whether a stronger, more useful notion to reduce the size of a set of partial implications could be based on 
partial implications following logically from several others together, beyond the single-premise case.

Simply considering standard examples with full implications like Augmentation (from $X \Rightarrow Y$ and $X^{\prime} \Rightarrow Y^{\prime}$ it follows $X X^{\prime} \Rightarrow Y Y^{\prime}$ ) or Transitivity (from $X \Rightarrow Y$ and $Y \Rightarrow Z$ it follows $X \Rightarrow Z$ ), it is easy to see that these cases fail badly for partial implications. Indeed, one might suspect, as this author did for quite some time, that one partial implication would not follow logically from several premises unless it follows from one of them.

Generally speaking, however, this suspicion is wrong. It is indeed true for confidence thresholds $\gamma \in(0,0.5)$, but these are not very useful in practice, as an association rule $X \rightarrow A$ of confidence less than 0.5 means that, in $\mathcal{D}_{X}$, the absence of $A$ is more frequent than its presence.

And, for $\gamma \in[0.5,1)$, it turns out that, for instance, from $A \rightarrow B C$ and $A \rightarrow B D$ it follows $A C D \rightarrow B$, in the sense that if both premises have confidence at least $\gamma$ in any dataset, then the conclusion also does. The general case for two premises was fully characterized in [7], but the case of arbitrary premise sets has remained elusive for some years. Eventually, a very recent result from [5] proved that redundancy with respect to a set of premises that are partial implications hinges on a complicated combinatorial property of the premises themselves. We give that property a short (if admittedly uninformative) name here:

Definition 2 Let $X_{1} \rightarrow Y_{1}, \ldots, X_{k} \rightarrow Y_{k}$ be a set of partial implications. We say that it is nice if $X_{1} \Rightarrow Y_{1}, \ldots, X_{k} \Rightarrow Y_{k} \models X_{i} \Rightarrow U$, for all $i \in 1 \ldots k$, where $U=X_{1} Y_{1} \cdots X_{k} Y_{k}$.

Here we use the standard symbol $\models$ for logical entailment; that is, whenever the implications at the left-hand side are true, the one at the right-hand side must be as well.

Note that the definition of nicety of a set of partial implications states a property, not of the partial implications themselves, but of their full counterparts. Then, we can characterize entailment among partial implications for high enough thresholds of confidence, as follows:

Theorem 1 [5] Let $X_{1} \rightarrow Y_{1}, \ldots, X_{k} \rightarrow Y_{k}$ be a set of partial implications with $k \geq 1$, candidates to premises, and a candidate conclusion $X_{0} \rightarrow Y_{0}$. If $\gamma \geq(k-1) / k$, then the following are equivalent:

1. in any dataset where the confidence of the premises $X_{1} \rightarrow Y_{1}, \ldots, X_{k} \rightarrow$ $Y_{k}$ is at least $\gamma, c\left(X_{0} \rightarrow Y_{0}\right) \geq \gamma$ as well; 
2. either $Y_{0} \subseteq X_{0}$, or there is a non-empty $L \subseteq\{1 \ldots k\}$ such that the following conditions hold:

(a) $\left\{X_{i} \rightarrow Y_{i}: i \in L\right\}$ is nice,

(b) $\bigcup_{i \in L} X_{i} \subseteq X_{0} \subseteq \bigcup_{i \in L} X_{i} Y_{i}$,

(c) $Y_{0} \subseteq X_{0} \cup \bigcap_{i \in L} Y_{i}$.

Interestingly, the last couple of conditions are reasonably correlated, for the case of several premises, with the central intuition that smaller antecedents are better than larger ones, and larger consequents are better than smaller ones. The premises actually necessary must all include the consequent of the conclusion, and their antecedents are to be included in the antecedent of the conclusion. Even the additional fact that the antecedent of the conclusion does not have "extra items" not present in the premises also makes sense.

However, there is the additional condition that only nice sets of partial implications may have a nontrivial logical consequence, and all this just for high enough confidence thresholds. The proof is complex and we refrain from discussing it here; see [5], where, additionally, the case of $\gamma<1 / k$ is also characterized and the pretty complicated picture for intermediate values of $\gamma$ is discussed.

We do indicate, though, that the notion of "nicety", in practice, turns out to be so restrictive that we have not found any case of nontrivial entailment from more than one premise in a number of tests with stardard benchmark datasets. Therefore, this approach is not particularly useful in practice to reduce the size of the outcome of an associator.

\subsection{Ongoing Developments}

As for representative rules (Subsection 3.2 ), there exists a natural variant of the question of redundancy, whereby full implications are handled separately; essentially, the redundancy notion becomes "closure-based". This extension was fully characterized as well for the case of two premises in [7], but it is current work in progress how to extend the scheme to the case of arbitrary quantities of premises. 


\section{Alternative Evaluation Measures}

We move on to discuss how to reinterpret the central intuition as we change the semantics of the partial implication connective. Confidence is widely used as a definition of partial implication but, in practice, presents two drawbacks. First, it does not detect negative correlations; and, second, as already indicated, often lets pass far too many rules and, moreover, fiddling with the confidence threshold turns out to be a mediocre or just useless solution. Examples of both disadvantages are both easy to construct and easy to find on popular benchmark datasets. Both objections can be addressed by changing the semantics of the expression $X \rightarrow Y$, by either replacing the confidence measure or by strengthening it with extra conditions. The literature on this topic is huge and cannot be reviewed here: see [16, 25, 35] and their references for information about the relevant developments published along these issues. We focus here on just a tiny subset of all these studies.

The first objection alluded to in the previous paragraph can be naturally solved via an extra normalization (more precisely, dividing the confidence by the support of the consequent). The outcome is lift, a well-known expression in basic probability; a closely related parameter is leverage:

Definition 3 Assume $X \cap Y=\emptyset$. The lift of partial implication $X \rightarrow Y$ is $\ell_{\mathcal{D}}(X \rightarrow Y)=\frac{c_{\mathcal{D}}(X \rightarrow Y)}{s_{\mathcal{D}}(Y)}=\frac{s_{\mathcal{D}}(X Y)}{s_{\mathcal{D}}(X) \times s_{\mathcal{D}}(Y)}$. The leverage of partial implication $X \rightarrow Y$ is $\lambda_{\mathcal{D}}(X \rightarrow Y)=s_{\mathcal{D}}(X Y)-s_{\mathcal{D}}(X) \times s_{\mathcal{D}}(Y)$.

If supports are unnormalized, extra factors $n$ are necessary. In case of independence of both sides of a partial implication $X \rightarrow Y$, we would have $s(X Y)=s(X) s(Y)$; therefore, both lift and leverage are measuring deviation from independence: lift is the multiplicative deviation, whereas leverage measures it rather as an additive distance instead. Leverage was introduced in [32] and, under the name "Novelty", in [24], and received much attention via the Magnum Opus associator [38]. We find lift in the references going by several different names: it has been called interest [34] or, in a slightly different but fully equivalent form, strength [33]; lift seems to be catching up as a short name, possibly aided by the fact that the Intelligent Miner system from IBM employed that name. These notions allow us to exemplify that we are modifying the semantics of our expressions: if we define the meaning of $X \rightarrow Y$ through confidence, then partial implications of the form $X \rightarrow Y$ and $X \rightarrow X Y$ are always equivalent, whereas, if we use lift, then they may 
not be. Note that, in case $X=\emptyset$, the lift trivializes to 1 . Also, if we are to use lift, then we must be careful to keep the right-hand side $Y$ disjoint from the left-hand side: $X \cap Y=\emptyset$.

A related notion is:

Definition 4 [24] The relative confidence of partial implication $X \rightarrow Y$, also called centered confidence or relative accuracy, is $r_{\mathcal{D}}(X \rightarrow Y)=c_{\mathcal{D}}(X \rightarrow$ $Y)-c_{\mathcal{D}}(\emptyset \rightarrow Y)$.

Therefore, the relative confidence is measuring additively the effect, on the support of the consequent $Y$, of "adding the condition" or antecedent $X$. Since $c_{\mathcal{D}}(\emptyset \rightarrow Y)=s_{\mathcal{D}}(Y)$, lift can be seen as comparing $c_{\mathcal{D}}(X \rightarrow Y)$ with $c_{\mathcal{D}}(\emptyset \rightarrow Y)$, that is, effecting the same comparison but multiplicatively this time: $\ell(X \rightarrow Y)=\frac{s(X Y)}{s(X) \times s(Y)}=\frac{c(X \rightarrow Y)}{s(Y)}=\frac{c(X \rightarrow Y)}{c(\emptyset \rightarrow Y)}$. Also, it is easy to check that leverage can be rewritten as $\lambda_{\mathcal{D}}(X \rightarrow Y)=s_{\mathcal{D}}(X) \times r_{\mathcal{D}}(X \rightarrow Y)$ and is therefore called also weighted relative accuracy [24]. Relative confidence has the potential to solve the "negative correlation" objection to confidence, and all subsequent measures to be described here inherit this property as well.

An objection of a different sort is that lift and leverage are symmetric. As the implicational syntax is asymmetric, they do not fit very well the directional intuition of an expression like $X \rightarrow Y$; that is one of the reasons behind the exploration of many other options. However, to date, none of the more sophisticate attempts seems to have gained a really noticeable "market share". Most common implementations either offer a long list of options of measures for the user to choose from (like [13] for one), or employ the simpler notions of confidence, support, lift, or leverage (for instance, Magnum Opus [38]). We believe that one must keep close to confidence and to deviation from independence. Confidence is the most natural option for many educated domain experts not specialized in data mining, and it provides actually a directionality to our partial implications.

The vast majority of these alternatives attempt at defining the quality of partial implication $X \rightarrow Y$ relying only on the supports of $X, Y, X Y$, or their complements. One major exception is improvement [12], which is the added confidence obtained by using the given antecedent as opposed to any properly smaller one. We discuss it and two other related quantities next. They are motivated again by our central intuition: if the confidence of a partial implication with a smaller antecedent and the same consequent is sufficiently high, the larger partial implication should not be provided in the 
output. They have in common that their computation requires exploration of a larger space, however; we return to this point in the next section.

\subsection{Improvement: Additive and Multiplicative}

The key observation for this section is that $X \rightarrow Y$ and $Z \rightarrow Y$, for $Z \subset X$, provide different, independent information. From the perspective of confidence, either may have it arbitrarily higher than the other. For inequality in one direction, suppose that almost all transactions with $X$ have $Y$, but they are just a small fraction of those supporting $Z$, which mostly lack $Y$; conversely, $Y$ might hold for most transactions having $Z$, but the only transactions having all of $X$ can be those without $Y$. In Example 1, one can see that $c(\emptyset \rightarrow B C)<c(A \rightarrow B C)$ whereas $c(\emptyset \rightarrow C)>c(B \rightarrow C)$.

This fact underlies the difficulty in choosing a proper confidence bound. Assume that there exists a mild correlation giving, say, $c(Z \rightarrow A)=2 / 3$. If the threshold is set higher, of course this rule is not found; but an undesirable side effect may appear: there may be many ways of choosing subsets of the support of $Z$, by enlarging it a bit, where $Y$ is frequent enough to pass the threshold. Thus, often, in practice, the algorithms enlarge $Z$ into various supersets $X_{i}$ so that all the confidences $c\left(X_{i} \rightarrow A\right)$ do pass, and then $Z \rightarrow A$ is not seen, but generates dozens of very similar "noisy" rules, to be manually explored and filtered. Finding the appropriate threshold becomes difficult, also because, for different partial implications, this sort of phenomenon may appear at several threshold values simultaneously.

Relative confidence tests confidence by a comparison to what happens if the antecedent is replaced by one of its subsets in particular, namely $\emptyset$. Improvement generalizes it by considering not only the alternative partial implication $\emptyset \rightarrow Y$ but all proper subsets of the antecedent, as alternative antecedents, and in the same additive form:

Definition 5 The improvement $X \rightarrow Y$, where $X \neq \emptyset$, is $i(X \rightarrow Y)=$ $\min \{c(X \rightarrow Y)-c(Z \rightarrow Y) \mid Z \subset X\}$.

The definition is due to [12], where only association rules are considered, that is, cases where $|Y|=1$. The work on productive rules [39] is related: these coincide with the rules of positive improvement. In [26], improvement is combined with further pruning on the basis of the $\chi^{2}$ value. We literally quote from [12]: "A rule with negative improvement is typically undesirable 
because the rule can be simplified to yield a proper sub-rule that is more predictive, and applies to an equal or larger population due to the antecedent containment relationship. An improvement greater than 0 is thus a desirable constraint in almost any application of association rule mining. A larger minimum on improvement is also often justified because most rules in dense data-sets are not useful due to conditions or combinations of conditions that add only a marginal increase in confidence."

The same process, and with the same intuitive justification, can be applied to lift, which is, actually, a multiplicative, instead of additive, version of relative confidence as indicated above: $\ell(X \rightarrow Y)=c(X \rightarrow Y) / c(\emptyset \rightarrow Y)$. Taking inspiration in this correspondence, we studied in [9] a multiplicative variant of improvement that generalizes lift, exactly in the same way as improvement generalizes relative confidence:

Definition 6 The multiplicative improvement of $X \rightarrow Y$, where $X \neq \emptyset$, is $m(X \rightarrow Y)=\min \{c(X \rightarrow Y) / c(Z \rightarrow Y) \mid Z \subset X\}$.

In Example 1, the facts that $c(A \rightarrow B C)=4 / 5$ and $c(\emptyset \rightarrow B C)=3 / 4$ lead to $i(A \rightarrow B C)=4 / 5-3 / 4=0.05$ and $m(A \rightarrow B C)=(4 / 5) /(3 / 4) \approx$ 1.066. Here, as the size of the antecedent is 1 , there is one single candidate $Z=\emptyset$ to proper subset of the antecedent and, therefore, improvement coincides with relative confidence, and multiplicative improvement coincides with lift. For larger left-hand sides, the values will be different in general.

\section{$5.2 \quad$ Rule Blocking}

Attempting at formalizing the same part of the central intuition, we proposed in [6] a notion of "rule blocking", where a smaller antecedent $Z \subset X$ would "block" (that is, suggest to omit) a given partial implication $X \rightarrow Y$. We will compare the number of tuples having $X Y$ (that is, having $Y$ within the supporting set of $X$ ) with the quantity that would be predicted from the confidence of the partial implication $Z \rightarrow Y$, that applies to a larger supporting set: we are going to bound the relative error incurred if the support $s(X)$ and the confidence of $Z \rightarrow Y$ are employed to approximate the confidence of $X \rightarrow Y$.

More precisely, let $c(Z \rightarrow Z Y)=c$. If $Y$ is distributed along the support of $X$ at the same ratio as along the larger support of $Z$, we would expect $s(X Y) \approx c \times s(X)$ : we consider the relative error committed by $c \times s(X)$ 
used as an approximation to $s(X Y)$ and, if the error is low, we consider that $Z \rightarrow Y$ is sufficient information about $X \rightarrow Y$ and dispose of this last one.

Definition 7 [6] $Z \subset X$ blocks $X \rightarrow Y$ at blocking threshold $\epsilon$ when

$$
\frac{s(X Y)-c(Z \rightarrow Y) s(X)}{c(Z \rightarrow Y) s(X)} \leq \epsilon .
$$

In case the difference in the numerator is negative, it would mean that $s(X Y)$ is even lower than what $Z \rightarrow Y$ would suggest. If it is positive but the quotient is low, $c(Z \rightarrow Y) \times s(X)$ still suggests a good approximation to $c(X \rightarrow Y)$, and the larger partial implication $X \rightarrow Y$ does not bring high enough confidence to be considered besides $Z \rightarrow Y$, a simpler one: it remains blocked. But, if the quotient is larger, and this happens for all $Z$, then $X \rightarrow$ $Y$ becomes interesting since its confidence is higher enough than suggested by other partial implications of the form $Z \rightarrow Y$ for smaller antecedents $Z$. Of course, the higher the block threshold, the more demanding the constraint is. Note that, in the presence of a support threshold $\tau, s(Z Y) \geq s(X Y)>\tau$ or a similar inequality would be additionally required. The value $\epsilon$ is intended to take positive but small values, say around 0.2 or lower. In Example 1 , $\emptyset$ blocks $A \rightarrow B C$ at blocking threshold $1 / 15 \approx 0.066$.

Rule blocking relates to multiplicative improvement as follows:

Proposition 2 The smallest blocking threshold at which $X \rightarrow Y$ is blocked is $m(X \rightarrow Y)-1$.

Proof As everything around is finite, this is equivalent to proving that $Z \subset$ $X$ blocks $X \rightarrow Y$ at block threshold $\epsilon$ if and only if $\frac{c(X \rightarrow Y)}{c(Z \rightarrow Y)}-1 \leq \epsilon$, for all such $Z$. Starting from the definition of blocking, multiplying both sides of the inequality by $c(Z \rightarrow Y)$, separating the two terms of the left-hand side, replacing $s(X Y) / s(X)$ by its meaning, $c(X \rightarrow Y)$, and then solving first for $c(Z \rightarrow Y)$ and finally for $\epsilon$, we find the stated equivalence. All the algebraic manipulations are reversible.

\subsection{Ongoing: Conditional Weighted Versions of Lift and Leverage}

We propose here one additional step to enhance the flexibility of both lift and leverage by considering their action, on the same partial implication, but 
with respect to many different subsets of the dataset, and under a weighting scheme that leads to different existing measures according to the weights chosen.

For a given partial implication $X \rightarrow Y$, we consider many limited views of the dataset, namely, all its projections into subsets of the antecedent. We propose to measure a weighted variant of the lift and/or the leverage of the same partial implication in all these projections, and evaluate as the quality of the partial implication the minimum value thus obtained. That is, we want our high-quality partial implications not only to have high lift or leverage, but also to maintain it when we consider projections of the dataset on the subsets of the antecedent. We call the measures obtained conditional weighted lift and leverage.

Definition 8 Assume $X \cap Y=\emptyset$. Let $w$ be a weighting function associating a weight (either a positive real number or $\infty$ ) to each proper subset of $X$. The conditional weighted lift of partial implication $X \rightarrow Y$ is $\ell_{\mathcal{D}, w}^{\prime}(X \rightarrow$ $Y)=\min \left\{w(Z) \ell_{\mathcal{D}_{Z}}(X \rightarrow Y) \mid Z \subseteq X\right\}$. The conditional weighted leverage of partial implication $X \rightarrow Y$ is $\lambda_{\mathcal{D}, w}^{\prime}(X \rightarrow Y)=\min \left\{w(Z) g_{\mathcal{D}_{Z}}(X \rightarrow Y) \mid\right.$ $Z \subseteq X\}$

These notions can be connected to other existing notions with unificatory effects. We only state here one such connection. Further development will be provided in a future paper in preparation.

Proposition 3 For inverse confidence weights, conditional weighted leverage is improvement: for all $X \rightarrow Y, \lambda_{\mathcal{D}, w}^{\prime}(X \rightarrow Y)=i(X \rightarrow Y)$ holds for the weighting function $w_{r}(Z)=c_{\mathcal{D}}(Z \rightarrow X)^{-1}$.

\section{Support Ratio and Confidence Boost}

From the perspective of our central intuition, the previous section has developed, essentially issues related to smallish antecedents. This is fully appropriate for the discussion of association rules, which were defined originally as partial implications with singleton consequents. We now briefly concentrate on largish consequents, and then join both perspectives. 


\subsection{Support ratio}

The support ratio was employed first, to our knowledge, in [23], where no particular name was assigned to it. Together with other similar quotients, it was introduced in order to help obtaining faster algorithmics.

Definition 9 In the presence of a support threshold $\tau$, the support ratio of a partial implication $X \rightarrow Y$ is

$$
\sigma(X \rightarrow Y)=\frac{s(X Y)}{\max \{s(Z) \mid X Y \subset Z, s(Z)>\tau\}}
$$

We see that this quantity depends on $X Y$ but not on the antecedent $X$ itself. In Example 1, we find that $\sigma(A \rightarrow B C)=4 / 3$.

\subsection{Confidence Boost}

Definition 10 The confidence boost of a partial implication $X \rightarrow Y$ (always with $X \cap Y=\emptyset)$ is $\beta(X \rightarrow Y)=$

$$
\frac{c(X \rightarrow X Y)}{\max \left\{c\left(X^{\prime} \rightarrow X^{\prime} Y^{\prime}\right) \mid(X \rightarrow X Y) \not \equiv\left(X^{\prime} \rightarrow X^{\prime} Y^{\prime}\right), X^{\prime} \subseteq X, Y \subseteq Y^{\prime}\right\}} .
$$

where the partial implications in the denominator are implicitly required to clear the support threshold, in case one is enforced: $s\left(X^{\prime} \rightarrow X^{\prime} Y^{\prime}\right)>\tau$.

Let us explain the interpretation of this parameter. Suppose that $\beta(X \rightarrow$ $Y)$ is low, say $\beta(X \rightarrow Y) \leq b$, where $b$ is just slightly larger than 1 . Then, according to the definition, there must exist some different partial implication $X^{\prime} \rightarrow X^{\prime} Y^{\prime}$, with $X^{\prime} \subseteq X$ and $Y \subseteq X^{\prime} Y^{\prime}$, such that $\frac{c(X \rightarrow Y)}{c\left(X^{\prime} \rightarrow Y^{\prime}\right)} \leq b$, or $c\left(X^{\prime} \rightarrow Y^{\prime}\right) \geq c(X \rightarrow Y) / b$. This inequality says that the partial implication $X^{\prime} \rightarrow Y^{\prime}$, stating that transactions with $X^{\prime}$ tend to have $X^{\prime} Y^{\prime}$, has a confidence relatively high, not much lower than that of $X \rightarrow Y$; equivalently, the confidence of $X \rightarrow Y$ is not much higher (it could be lower) than that of $X^{\prime} \rightarrow Y^{\prime}$. But all transactions having $X$ do have $X^{\prime}$, and all transactions having $Y^{\prime}$ have $Y$, so that the confidence found for $X \rightarrow Y$ is not really that novel, given that it does not give so much additional confidence over a partial implication that states such a similarly confident, and intuitively stronger, fact, namely $X^{\prime} \rightarrow Y^{\prime}$. 
This author has developed a quite successful open-source partial implication miner based on confidence boost (yacaree.sf.net); all readers are welcome to experiment with it and provide feedback. We note also that the confidence width alluded to in Section 3.1, while having different theoretical and practical properties, is surprisingly close in definition to confidence boost. See [8] for further discussion of all these issues. Confidence boost fits the general picture as follows:

Proposition $4 \beta(X \rightarrow Y)=\min \{\sigma(X \rightarrow Y), m(X \rightarrow Y)\}$.

The inequalities $\beta(X \rightarrow Y) \leq \sigma(X \rightarrow Y)$ (due to [11]) and $\beta(X \rightarrow Y) \leq$ $m(X \rightarrow Y)$ are simple to argue: the consequent leading to the support ratio, or the antecedent leading to the multiplicative improvement, take a role in the denominator of confidence boost. Conversely, taking the maximizing partial implication in the denominator, if it has the same antecedent $X$ then one obtains a bound on the support ratio whereas, if the antecedent is properly smaller, a bound on the multiplicative improvement follows.

In Example 1, since $\sigma(A \rightarrow B C)=4 / 3$ and $m(A \rightarrow B C)=(4 / 5) /(3 / 4)$, which is smaller, we obtain $\beta(A \rightarrow B C)=(4 / 5) /(3 / 4) \approx 1.066$.

A related proposal in [22] suggests to minimize directly the antecedents and maximizing the consequents, within the confidence bound, and in a context where antecedents and consequents are kept disjoint. This is similar to statement (3) in Lemma 1, except that, there, one maximizes jointly consequent and antecedent. If consequents are maximized separately, then the central intuition fails, but there is an interesting connection with confidence boost; see [8].

The measures in this family of improvement, including conditional weighted variants and also confidence boost, tend to require exploration of larger spaces of antecedents compared to simpler rule quality measures. This objection turns out not to be too relevant because human-readable partial implications have often just a few items in the antecedent. Nontrivial algorithmic proposals for handling this issue appear as well in [8].

\subsection{Ongoing Developments}

We briefly mention here the following observations. First, like in Section 3.2, a variant of confidence boost appropriate for closure-based analysis exists [8]. Second, both variants trivialize if they are applied directly, in their literal 
terms, to full implications. However, the intuitions leading to confidence boost can be applied as well to full implications. In future work, currently in preparation, we will discuss proposals for formalizing the same intuition in the context of full implications.

\section{Evaluation of Evaluation Measures}

We have covered just a small fraction of the evaluation measures proposed to endow with useful semantics the partial implication connective. All of those attempt, actually, at capturing a potential (but maybe nonexisting) "naïve concept" of interesting partial implication from the perspective of an end user. Eventually, we would like to find one such semantics that fits as best as possible that hypothetical naïve concept.

We can see no choice but to embark, at some point, in the creation of resources where, for specific datasets, the interest of particular implications is recorded as per the assessment of individual humans. Some approximations to this plan are Section 5.2 of [8], where the author, as a scientific expert, subjectively evaluates partial implications obtained from abstracts or scientific papers; a similar approach in [14] using PKDD abstracts; and the work in [10, 44] where partial implications found on educational datasets from university course logs are evaluated by the teachers of the corresponding courses. These preliminary experiments are positive and we hope that a more ambitious attempt could be made in the future along these lines.

The idea of evaluating associators through the predictive capabilities of the rules found has been put forward in several sources, e.g. [29]. The usage of association rules for direct prediction (where the "class" attribute is forced to occur in the consequent) has been widely studied (e.g. [41]). In [29], two different associators are employed to find rules with the "class"

as consequent, and they are compared in terms of predictive accuracy. This scheme is inappropriate to evaluate our proposals for the semantics of partial implications, because, first, we must focus on single pairs of attribute and value as right-hand side, thus making it useless to consider larger right-hand sides; and, also, the classification will only be sensible to minimal left-hand sides independently of their confidences.

In [9], we have deployed an alternative framework that allows us to evaluate the diverse options of semantics for association rules, in terms of their usefulnes for subsequent predictive tasks. By means of a mechanism akin 
to the AUC measure for predictor evaluation, we have focused on potential accuracy improvements of predictors on given, public, standard benchmark datasets, if one more Boolean column is added, namely, one that is true exactly for those observations that are exceptions to one association rule: the antecedent holds but the consequent does not. In a sense, we use the association rule as a "hint of outliers", but, instead of removing them, we simply offer direct access to this label to the predictor, through the extra column. Of course, in general this may lead astray the predictor instead of helping it. Our experiments suggest that leverage, support, and multiplicative improvement tend to be better than the other measures with respect to this evaluation score.

\subsection{Ongoing Developments}

We are currently developing yet new frameworks that, hopefully, might be helpful in assessing the relative merits of the different candidates for semantics of partial implications, put forward often as rule quality measures. One of them resorts to an empirical application of approximations to the MDL principle along the lines of Krimp [37]. A second idea is to make explicit the dependence on alternative partial implications, in the sense that $X \rightarrow Y$ would mean, intuitively, that $Y$ appears often on the support of $X$ and that, barring the presence of some other partial implication to the contrary, it is approximately uniformly distributed there. These avenues will be hopefully explored along the coming months or years. A common thread is that additional statistical knowledge, along the lines of the self-sufficient itemsets of Webb [40], for instance, is expected to be at play in the future developments of the issue of endowing the partial implication connective with the right intuitive semantics.

\section{References}

[1] C. C. Aggarwal and J. Han, editors. Frequent Pattern Mining. Springer, 2014.

[2] C. C. Aggarwal and P. S. Yu. A new approach to online generation of association rules. IEEE Transactions on Knowledge and Data Engineering, 13(4):527-540, 2001. 
[3] R. Agrawal, T. Imielinski, and A. N. Swami. Mining association rules between sets of items in large databases. In P. Buneman and S. Jajodia, editors, SIGMOD Conference, pages 207-216. ACM Press, 1993.

[4] R. Agrawal, H. Mannila, R. Srikant, H. Toivonen, and A. I. Verkamo. Fast discovery of association rules. In Advances in Knowledge Discovery and Data Mining, pages 307-328. AAAI/MIT Press, 1996.

[5] A. Atserias and J. L. Balcázar. Entailment among probabilistic implications. Accepted for presentation at LICS, 2015.

[6] J. L. Balcázar. Two measures of objective novelty in association rule mining. In PAKDD Workshops (Springer-Verlag LNCS 5669), pages 76-98, 2009.

[7] J. L. Balcázar. Redundancy, deduction schemes, and minimum-size bases for association rules. Logical Methods in Computer Science, $6(2: 3): 1-33,2010$.

[8] J. L. Balcázar. Formal and computational properties of the confidence boost of association rules. TKDD, 7(4):19, 2013.

[9] J. L. Balcázar and F. Dogbey. Evaluation of association rule quality measures through feature extraction. In A. Tucker, F. Höppner, A. Siebes, and S. Swift, editors, Advances in Intelligent Data Analysis XII - 12th International Symposium, IDA 2013, London, UK, October 17-19, 2013. Proceedings, volume 8207 of Lecture Notes in Computer Science, pages 68-79. Springer, 2013.

[10] J. L. Balcázar, C. Tîrnăucă, and M. Zorrilla. Mining educational data for patterns with negations and high confidence boost. Taller de Minería de Datos TAMIDA 2010; available at: http://personales.unican.es/tirnaucac 2010.

[11] J. L. Balcázar, C. Tîrnăucă, and M. E. Zorrilla. Filtering association rules with negations on the basis of their confidence boost. KDIR 2010. Available at: http://personales.unican.es/tirnaucac , 2010.

[12] R. Bayardo, R. Agrawal, and D. Gunopulos. Constraint-based rule mining in large, dense databases. In ICDE, pages 188-197, 1999. 
[13] C. Borgelt. Efficient implementations of Apriori and Eclat. In B. Goethals and M. J. Zaki, editors, FIMI, volume 90 of CEUR Workshop Proceedings. CEUR-WS.org, 2003.

[14] A. Gallo, T. De Bie, and N. Cristianini. Mini: Mining informative nonredundant itemsets. In J. N. Kok, J. Koronacki, R. L. de Mántaras, S. Matwin, D. Mladenic, and A. Skowron, editors, PKDD, volume 4702 of Lecture Notes in Computer Science, pages 438-445. Springer, 2007.

[15] B. Ganter and R. Wille. Formal Concept Analysis: Mathematical Foundations. Springer-Verlag, 1999.

[16] L. Geng and H. J. Hamilton. Interestingness measures for data mining: A survey. ACM Comput. Surv., 38(3), 2006.

[17] J. Guigues and V. Duquenne. Familles minimales d'implications informatives resultants d'un tableau de données binaires. Math. Sci. Hum., 95:5-18, 1986.

[18] P. Hájek, M. Holeňa, and J. Rauch. The GUHA method and its meaning for data mining. J. Comput. Syst. Sci., 76(1):34-48, 2010.

[19] J. Han, J. Pei, Y. Yin, and R. Mao. Mining frequent patterns without candidate generation: A frequent-pattern tree approach. Data Min. Knowl. Discov., 8(1):53-87, 2004.

[20] S. Jaroszewicz, T. Scheffer, and D. A. Simovici. Scalable pattern mining with bayesian networks as background knowledge. Data Min. Knowl. Discov., 18(1):56-100, 2009.

[21] M. Kryszkiewicz. Representative association rules. In X. Wu, K. Ramamohanarao, and K. B. Korb, editors, Proc. of the 2nd Pacific-Asia Conference on Knowledge Discovery and Data Mining (PAKDD), volume 1394 of Lecture Notes in Artificial Intelligence, pages 198-209. Springer-Verlag, 1998.

[22] M. Kryszkiewicz. Representative association rules and minimum condition maximum consequence association rules. In J. M. Zytkow and M. Quafafou, editors, PKDD, volume 1510 of Lecture Notes in Computer Science, pages 361-369. Springer, 1998. 
[23] M. Kryszkiewicz. Closed set based discovery of representative association rules. In F. Hoffmann, D. J. Hand, N. M. Adams, D. H. Fisher, and G. Guimarães, editors, Proc. of the 4 th International Symposium on Intelligent Data Analysis (IDA), volume 2189 of Lecture Notes in Computer Science, pages 350-359. Springer-Verlag, 2001.

[24] N. Lavrac, P. A. Flach, and B. Zupan. Rule evaluation measures: A unifying view. In S. Dzeroski and P. A. Flach, editors, ILP, volume 1634 of Lecture Notes in Computer Science, pages 174-185. Springer, 1999.

[25] P. Lenca, P. Meyer, B. Vaillant, and S. Lallich. On selecting interestingness measures for association rules: User oriented description and multiple criteria decision aid. European Journal of Operational Research, 184(2):610-626, 2008.

[26] B. Liu, W. Hsu, and Y. Ma. Pruning and summarizing the discovered associations. In Proc. Knowledge Discovery in Databases, pages 125$134,1999$.

[27] M. Luxenburger. Implications partielles dans un contexte. Mathématiques et Sciences Humaines, 29:35-55, 1991.

[28] N. Megiddo and R. Srikant. Discovering predictive association rules. In Proc. Knowledge Discovery in Databases, pages 274-278, 1998.

[29] S. Mutter, M. Hall, and E. Frank. Using classification to evaluate the output of confidence-based association rule mining. In G. I. Webb and X. Yu, editors, Australian Conference on Artificial Intelligence, volume 3339 of Lecture Notes in Computer Science, pages 538-549. Springer, 2004.

[30] B. Padmanabhan and A. Tuzhilin. Small is beautiful: discovering the minimal set of unexpected patterns. In Proc. Knowledge Discovery in Databases, pages 54-63, 2000.

[31] V. Phan-Luong. The representative basis for association rules. In N. Cercone, T. Y. Lin, and X. Wu, editors, Proc. of the 2001 IEEE International Conference on Data Mining (ICDM), pages 639-640. IEEE Computer Society, 2001. 
[32] G. Piatetsky-Shapiro. Discovery, analysis, and presentation of strong rules. In Proc. Knowledge Discovery in Databases, pages 229-248, 1991.

[33] D. Shah, L. Lakshmanan, K. Ramamritham, and S. Sudarshan. Interestingness and pruning of mined patterns. In ACM SIGMOD Workshop on Research Issues in Data Mining and Knowledge Discovery, 1999.

[34] C. Silverstein, S. Brin, and R. Motwani. Beyond market baskets: Generalizing association rules to dependence rules. Data Min. Knowl. Discov., 2(1):39-68, 1998.

[35] P.-N. Tan, V. Kumar, and J. Srivastava. Selecting the right objective measure for association analysis. Information Systems, 29(4):293-313, 2004.

[36] H. Toivonen, M. Klemettinen, P. Ronkainen, K. Hätönen, and H. Mannila. Pruning and grouping discovered association rules. In ECML-95 Workshop on Statistics, Machine Learning, and Knowledge Discovery in Databases, pages 47-52, 1995.

[37] J. Vreeken, M. van Leeuwen, and A. Siebes. Krimp: mining itemsets that compress. Data Min. Knowl. Discov., 23(1):169-214, 2011.

[38] G. I. Webb. Efficient search for association rules. In R. Ramakrishnan, S. J. Stolfo, R. J. Bayardo, and I. Parsa, editors, Proceedings of the sixth ACM SIGKDD international conference on Knowledge discovery and data mining, Boston, MA, USA, August 20-23, 2000, pages 99-107. ACM, 2000.

[39] G. I. Webb. Discovering significant patterns. Machine Learning, 68(1):133, 2007.

[40] G. I. Webb. Self-sufficient itemsets: An approach to screening potentially interesting associations between items. TKDD, 4(1), 2010.

[41] X. Yin and J. Han. CPAR: classification based on predictive association rules. In D. Barbará and C. Kamath, editors, Proceedings of the Third SIAM International Conference on Data Mining, San Francisco, CA, USA, May 1-3, 2003, pages 331-335. SIAM, 2003. 
[42] M. J. Zaki. Mining non-redundant association rules. Data Min. Knowl. Discov., 9(3):223-248, 2004.

[43] M. J. Zaki and J. Wagner Meira. Data Mining and Analysis: Fundamental Concepts and Algorithms. Cambridge University Press, May 2014.

[44] M. E. Zorrilla, D. García-Sáiz, and J. L. Balcázar. Towards parameterfree data mining: Mining educational data with yacaree. In M. Pechenizkiy, T. Calders, C. Conati, S. Ventura, C. Romero, and J. C. Stamper, editors, EDM, pages 363-364. www.educationaldatamining.org, 2011. 